\title{
Therapeutic Endoscopic Treatment Plus Maintenance Dimethyl Sulfoxide Therapy Prolongs Recurrence-Free Time in Patients With Hunner Type Interstitial Cystitis: A Pilot Study
}

\author{
Atsushi Otsuka, Takahisa Suzuki, Yuto Matsushita, Hiromitsu Watanabe, Keita Tamura, Daisuke Motoyama, Toshiki Ito, \\ Takayuki Sugiyama, Hideaki Miyake
}

Department of Urology, Hamamatsu University School of Medicine, Hamamatsu, Japan

Purpose: To evaluate whether hydrodistention with fulguration of Hunner lesions (HD/FUL) plus maintenance dimethyl sulfoxide (DMSO) therapy prolongs the recurrence-free time in patients with Hunner type interstitial cystitis (IC).

Methods: The study enrolled patients with Hunner type IC who required repeat HD/FUL due to recurrence of IC symptoms after the first HD/FUL at our institution. All patients received a second HD/FUL plus maintenance DMSO therapy. The maintenance DMSO therapy was performed every 2 weeks for a total of 8 instillations, and then once every 4 weeks thereafter. The recurrencefree time from HD/FUL to therapeutic failure was estimated using the Kaplan-Meier method. The recurrence-free time between the first $\mathrm{HD} / \mathrm{FUL}$ and second $\mathrm{HD} / \mathrm{FUL}$ plus maintenance DMSO therapy was statistically compared using the log-rank test.

Results: A total of 21 patients (mean age, $66.3 \pm 10.8$ years) with Hunner type IC were evaluated. The recurrence-free time for the second HD/FUL plus maintenance DMSO therapy was significantly longer than that for the first HD/FUL $(\mathrm{P}<0.0001)$. The median recurrence-free time for the first HD/FUL was 10.1 months, while that for the second HD/FUL plus maintenance DMSO therapy has yet to be reached. The recurrence-free rate for the first HD/FUL was $81.0 \%$ at 6 months, $38.1 \%$ at 1 year, $9.5 \%$ at 2 years, and $4.8 \%$ at 3 years. In contrast, the rate for the second HD/FUL plus maintenance DMSO therapy was $100 \%$ at 6 months, $94.7 \%$ at 1 year, $82.6 \%$ at 2 years, and $82.6 \%$ at 3 years. There were no significant differences in efficacy between the first and second HD/FUL.

Conclusions: HD/FUL plus maintenance DMSO therapy clearly prolongs the recurrence-free time compared with HD/FUL alone in Hunner type IC.

Keywords: Cystitis, interstitial; Dimethyl sulfoxide; Hydrodistention; Fulguration; Maintenance

- Research Ethics: This study was performed according to the Declaration of Helsinki and was approved by the ethics committee of Hamamatsu University School of Medicine (14-302). Written informed consent was obtained from all subjects.

- Conflict of Interest: No potential conflict of interest relevant to this article was reported.

\section{INTRODUCTION}

Interstitial cystitis (IC) is a disease of the urinary bladder with lower urinary tract symptoms such as urinary frequency, blad- der hypersensitivity and/or bladder pain. IC is often associated with serious impairment of quality of life [1]. Because its pathological mechanism is still unknown, curative therapy for IC has yet to be established.

Corresponding author: Atsushi Otsuka (iD https://orcid.org/0000-0001-6472-0988 Department of Urology, Hamamatsu University School of Medicine, 1-20-1 Handayama, Higashi-ku, Hamamatsu, Shizuoka 431-3192, Japan

E-mail: otsuka@hama-med.ac.jp / Tel: +81-53-435-2306 / Fax: +81-53-435-2305

Submitted: June 28, 2019 / Accepted after revision: November 8, 2019 
Hydrodistention is used to both diagnose IC and treat symptoms, including pain, urgency, and urinary frequency. The positive effects of hydrodistention last only about 6 months [2-4]. Fulguration of Hunner lesions is an effective treatment for Hunner type IC patients. However, approximately $50 \%$ of patients who undergo fulguration require a repeat procedure within a few years [5-8]. There is therefore a need for definitive therapy to prevent recurrence of IC symptoms following therapeutic endoscopic treatment. To resolve the short duration of endoscopic efficacy, studies have examined combination treatment with intravesical injections of botulinum toxin A, intravesical instillation of hyaluronic acid, or bladder training $[4,9,10]$.

Intravesical treatment for IC has been used for symptoms that are refractory or resistant to initial treatment, including conservative therapy and/or oral medications. For decades, intravesical instillation of dimethyl sulfoxide (DMSO) has been the cornerstone of IC treatment [11]. DMSO reportedly has local analgesic, anti-inflammatory, muscle-relaxing, and bacteriostatic effects [12-15]. Based on these mechanisms, we hypothesized that maintenance DMSO therapy following therapeutic endoscopic treatment may prolong the recurrence-free time compared to that achieved with therapeutic endoscopic treatment alone. However, few reports have evaluated the efficacy of maintenance DMSO therapy following therapeutic endoscopic treatment.

The aim of this study was to evaluate whether therapeutic endoscopic treatment (hydrodistention with fulguration of Hunner lesions, HD/FUL) plus maintenance DMSO therapy prolongs the recurrence-free time in patients with Hunner type IC.

\section{MATERIALS AND METHODS}

This prospective study (UMIN000027494) included patients with Hunner type IC who required a repeat HD/FUL due to the recurrence of IC symptoms after the first HD/FUL at our institution, despite all patients having received various other additional treatments with patient education and oral medications. The enrolled patients received the second HD/FUL plus maintenance DMSO therapy from August 2007 to March 2019. The protocol of this study was approved by the institutional ethics committee (14-302), and written informed consent was obtained from all patients.

Hunner type IC was defined as a disease of the urinary bladder meeting the following 3 criteria: (1) lower urinary tract symptoms such as urinary frequency, bladder hypersensitivity and/or bladder pain; (2) bladder pathology proven endoscopi- cally by the presence of Hunner lesions; and (3) exclusion of other diseases such as infection, malignancy, or calculi of the urinary tract [1]. The patients had undergone an initial evaluation before the first HD/FUL at our institution consisting of historytaking, physical examination, urinalysis, O'Leary-Sant Interstitial Cystitis Symptom and Problem Indices (ICSI and ICPI), visual analogue scale (VAS) for pain and urgency from $0-9(0$, none to 9, intolerable), and a 3-day bladder diary. If necessary, patients were evaluated using urine cytology, urine culture, transabdominal ultrasonography, computed tomography, or magnetic resonance imaging to exclude other diseases or conditions.

Hydrodistention was performed by overdistending the bladder using physiological saline at $80 \mathrm{~cm} \mathrm{H}_{2} \mathrm{O}$ with continuous endoscopic observation under general anaesthesia. At the early phase of filling with saline, Hunner lesions were carefully observed. After overdistention, pressure was maintained for 3 minutes. To avoid saline leakage around the cystoscope during the filling phase, the operators manually squeezed the urethra through the anterior vaginal wall in female patients. While the saline was continuously and slowly drained, the bladder mucosa was evaluated for bleeding. The drained volume was measured using a measuring cylinder. These procedures were repeated twice. Hunner lesions were electrically fulgurated after overdistending the bladder.

For the second HD/FUL, patients were subsequently started on maintenance DMSO therapy within one month (mean duration, 21.4 \pm 7.2 days). The protocol for this study is shown in Fig. 1. The maintenance DMSO therapy was provided every 2 weeks for a total of 8 instillations, and then once every 4 weeks thereafter. Intravesical instillation was performed using $50 \mathrm{~mL}$ of $50 \%$ DMSO, which was retained in the bladder for 15 minutes. Maintenance DMSO therapy was continued until the patient withdrew consent, IC symptoms requiring repeat endoscopic treatment or other intravesical instillation therapy recurred, or

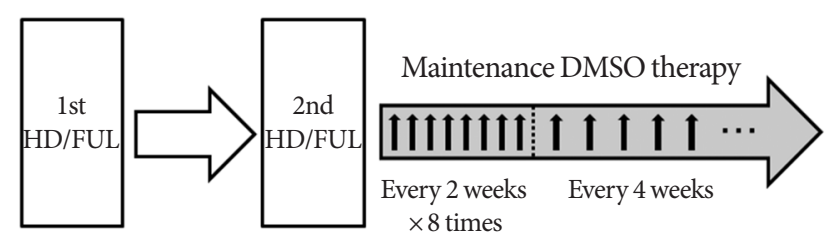

Patient education (stress reduction, fluid-intake, dietary intervention) Oral treatment (amitriptyline, hydroxyzine, suplatast tosilate, or NSAIDs)

Fig. 1. Study design. HD/FUL, hydrodistention with fulguration for Hunner lesions; DMSO, dimethyl sulfoxide; NSAIDs, nonsteroidal anti-inflammatory drugs. 
adverse events made it impossible to continue DMSO therapy. If patients needed to control temporary pain or urgency accompanying DMSO instillation, they were allowed to use oral or rectal nonsteroidal anti-inflammatory drugs (NSAIDs).

Therapeutic failure was defined as the need to receive repeat endoscopic treatment or other intravesical instillation therapy due to the recurrence of IC symptoms, despite receiving other additional treatments including patient education (instructions on stress reduction, fluid intake, and dietary interventions) and oral medications (amitriptyline, hydroxyzine, suplatast tosilate, or NSAIDs) to control IC symptoms as appropriate.

The recurrence-free time from HD/FUL to therapeutic failure was estimated using the Kaplan-Meier method. The recurrence-free time from the first HD/FUL to therapeutic failure was used as a control (intrasubject controlled analysis). The recurrence-free time between the first HD/FUL and second HD/ FUL plus maintenance DMSO therapy was statistically compared using the log-rank test. For patients with no therapeutic failure, their recurrence-free time was censored at the time of discontinuation of maintenance DMSO therapy or at the last visit, whichever was earlier.

In the first HD/FUL (control), the final assessment was performed at the time of recurrence of IC symptoms. In the second HD/FUL plus maintenance DMSO therapy, the final assessment was performed at the time of recurrence of IC symptoms, discontinuation of maintenance DMSO therapy, or at the last visit, whichever was earlier. All patients completed ICSI, ICPI, VAS for pain and urgency, and a bladder diary before and 1 month after HD/FUL, as well as at the final assessment. To rule out the possibility that a difference in efficacy between the first and second HD/FUL affected the efficacy of maintenance DMSO therapy, changes in clinical variables between pre- and post-HD/FUL were statistically compared using a t-test.

Statistical analyses were performed using IBM SPSS Statistics ver. 23.0 (IBM Co., Armonk, NY, USA) and GraphPad Prism 6 (GraphPad, San Diego, CA, USA). Statistical significance was defined as a P-value $<0.05$.

\section{RESULTS}

A total of 23 patients with Hunner type IC who required repeat

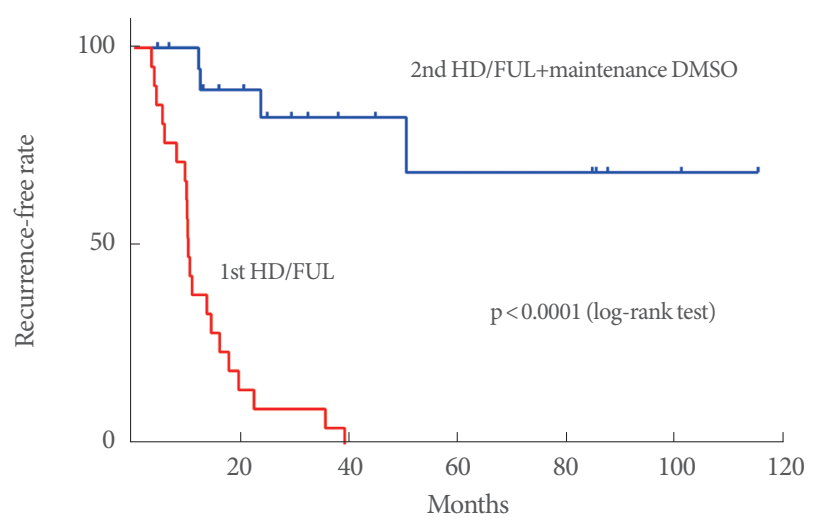

Fig. 2. Kaplan-Meier plots of recurrence-free time to therapeutic failure between the first HD/FUL and second HD/FUL plus maintenance DMSO therapy in Hunner type interstitial cystitis. HD/FUL, hydrodistention with fulguration for Hunner lesions; DMSO, dimethyl sulfoxide.

Table 1. Patient's characteristics before the first HD/FUL and the second HD/FUL plus maintenance DMSO $(n=21)$

\begin{tabular}{lccc}
\hline Characteristic & 1st HD/FUL & 2nd HD/FUL+DMSO & P-value \\
\hline ICSI & $16.7 \pm 3.6$ & $16.5 \pm 2.9$ & 0.818 \\
ICPI & $13.8 \pm 2.8$ & $13.6 \pm 2.5$ & 0.760 \\
VAS pain & $7.9 \pm 1.5$ & $7.5 \pm 1.7$ & 0.406 \\
VAS urgency & $7.8 \pm 1.8$ & $7.6 \pm 1.7$ & 0.673 \\
No. of micturitions/24hr & $18.3 \pm 7.6$ & $17.2 \pm 6.6$ & 0.440 \\
No. of nocturia episodes $/ 24 \mathrm{hr}$ & $4.5 \pm 2.3$ & $4.6 \pm 2.7$ & 0.826 \\
No. of urgency episodes $/ 24 \mathrm{hr}$ & $4.8 \pm 7.8$ & $2.8 \pm 5.4$ & 0.292 \\
Average voided volume $(\mathrm{mL})$ & $94 \pm 33$ & $101 \pm 44$ & 0.769 \\
Maximum voided volume $(\mathrm{mL})$ & $165 \pm 68$ & $158 \pm 73$ & 0.400
\end{tabular}

Values are presented as mean \pm standard deviation.

HD/FUL, hydrodistention with fulguration of Hunner lesions; DMSO, dimethyl sulfoxide; ICSI, Interstitial Cystitis Symptom Index; ICPI, Interstitial Cystitis Problem Index; VAS, visual analogue scale. 
HD/FUL due to the recurrence of IC symptoms after the first HD/FUL at our institution were enrolled from February 2007 to September 2018. After excluding 2 patients because of protocol violation and withdrawal of consent, 21 patients ( 3 males and 18 females) with Hunner type IC were evaluated. The mean age \pm standard deviation of the patients was $66.3 \pm 10.8$ years (range, 43-79 years). The mean duration from the first $\mathrm{HD} /$ FUL to the recurrence of IC symptoms was $13.2 \pm 9.4$ months. Eighteen patients (85.7\%) met the National Institute of Diabetes and Digestive and Kidney Diseases criteria [16]. Patients' characteristics before the first and second HD/FUL are shown in Table 1. There were no significant differences in any preoperative variables between the first and second HD/FUL. The mean duration of the follow-up from the start of maintenance DMSO therapy to the last visit was $71.8 \pm 39.8$ months.

Among the 21 patients, 10 patients continued maintenance DMSO therapy without therapeutic failure (median duration, 41 months; range, 7-114 months), and 4 patients experienced therapeutic failure (median duration, 18 months; range, 12-50 months). Although 7 patients discontinued maintenance DMSO therapy because of sustained remission of IC symptoms $(\mathrm{n}=4)$, urgency and pain accompanying DMSO instillation
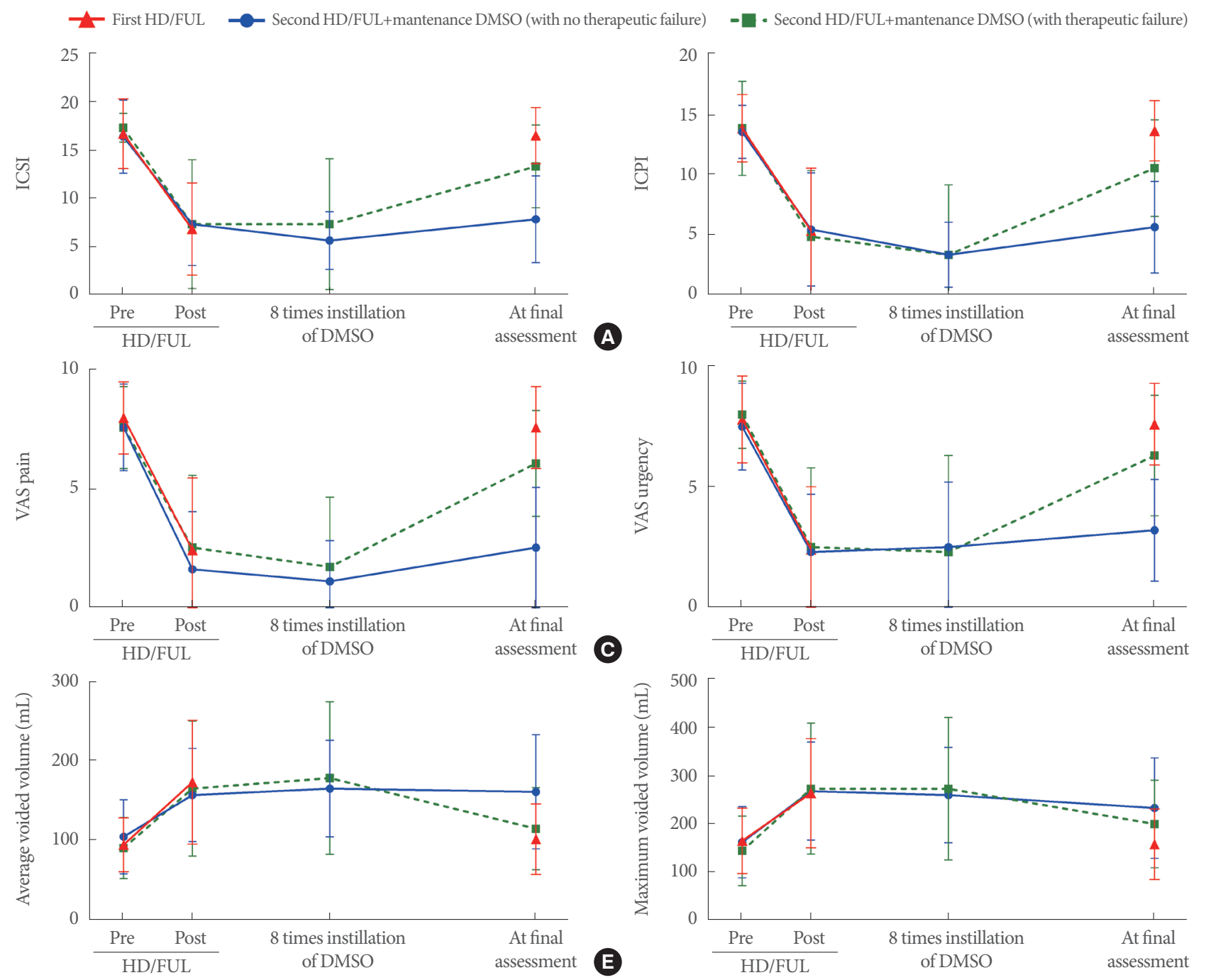

Fig. 3. Changes in ICSI (A), ICPI (B), VAS pain (C), VAS urgency (D), average voided volume (E), and maximum voided volume (F) at the first HD/FUL and second HD/FUL plus maintenance DMSO therapy (with or without therapeutic failure). ICSI, Interstitial Cystitis Symptom Index; ICPI, Interstitial Cystitis Problem Index; VAS, visual analogue scale; HD/FUL, hydrodistention with fulguration for Hunner lesions; DMSO, dimethyl sulfoxide. 
$(\mathrm{n}=1)$, loss to follow-up $(\mathrm{n}=1)$, and onset of idiopathic thrombocytopenia $(n=1)$, they did not experience therapeutic failure during the maintenance DMSO therapy (median duration. 23 months; range, 5-87 months).

Plots of the recurrence-free time for the first HD/FUL and second HD/FUL plus maintenance DMSO therapy are shown in Fig. 2. The log-rank test showed that the recurrence-free time for the second HD/FUL plus maintenance DMSO therapy was significantly longer than that for the first HD/FUL $(\mathrm{P}<0.0001)$. Median recurrence-free time for the first HD/FUL was 10.1 months, while that for the second HD/FUL plus maintenance DMSO therapy has yet to be reached. The recurrence-free rate for the first HD/FUL was $81.0 \%$ at 6 months, $38.1 \%$ at 1 year, $9.5 \%$ at 2 years, and $4.8 \%$ at 3 years. In contrast, the rate for the second HD/FUL plus maintenance DMSO therapy was $100 \%$ at 6 months, $94.7 \%$ at 1 year, $82.6 \%$ at 2 years, and $82.6 \%$ at 3 years.

Fig. 3 shows the changes in clinical variables of the first $\mathrm{HD} /$ FUL and second HD/FUL plus maintenance DMSO therapy (with and without therapeutic failure). There were no significant differences in efficacy between the first and second HD/ FUL. The maximum bladder capacity $(\mathrm{mL})$ at the first and second HD/FUL was $586 \pm 194$ and $521 \pm 185$, respectively $(\mathrm{P}=$ 0.268). Also, there was no significant difference in the number of Hunner lesions between the first and second HD/FUL ( $2.1 \pm 1.8$ vs. $2.4 \pm 1.4, \mathrm{P}=0.623$ ). Further, between patients with and without therapeutic failure of DMSO instillation, there were no significant differences of the maximum bladder capacity ( $475 \pm 95$ vs. $531 \pm 201)$ or the number of Hunner lesions (1.3 \pm 0.5 vs. $2.6 \pm 1.6)$ at the second HD/FUL.

\section{DISCUSSION}

Intravesical DMSO instillation is commonly used for the treatment of IC. Most studies have reported short- or middle-term efficacy of DMSO for patients with recurrent disease or who are refractory to conservative therapy. However, few studies have examined the efficacy of DMSO in IC patients stratified into Hunner type IC and non-Hunner type IC. Ek et al. [17] reported that, among 17 patients with Hunner lesions, DMSO improved pain and urinary frequency in 12 patients but did not improve bladder capacity. Peeker et al. [18] reported that while DMSO had no positive effect on maximal functional capacity, it significantly reduced pain and urinary frequency, but only in patients with classic IC (Hunner type IC). Recently, Tomoe [19] reported the effectiveness of maintenance DMSO therapy for 24 months fol- lowing therapeutic hydrodistention in Hunner type IC patients. She concluded that DMSO intravesical instillation therapy was useful for both maintaining and improving the effectiveness of hydrodistention in IC patients with Hunner lesions [19]. These studies demonstrate that, regardless of the presence or absence of pretreatment with hydrodistension, DMSO had short- or middle-term efficacy for treating Hunner type IC. However, from a recurrence prevention perspective, there have been no reports on the long-term efficacy of maintenance DMSO therapy following therapeutic endoscopic treatment.

The hallmark characteristic of IC is Hunner lesions. Fulguration of Hunner lesions is an effective treatment for ameliorating IC symptoms [5-8]. The American Urological Association guidelines recommend performing fulguration with a laser or electrocautery for Hunner lesions [20,21]. Further, Lee et al. [22] recently reported that transurethral resection of Hunner lesions combined with therapeutic hydrodistention increases bladder capacity and improves voiding symptoms more so than transurethral resection alone for ulcerative IC. Regrettably, even with concomitant therapeutic hydrodistention, approximately $50 \%$ of patients who undergo fulguration require a repeat procedure within a few years $[7,17]$. This evidence demonstrates the need for an effective treatment to prolong the recurrence-free time following hydrodistention with fulguration. The present study clearly demonstrates that maintenance DMSO therapy may be the novel therapeutic approach needed to prolong recurrence-free time following therapeutic endoscopic treatment in Hunner type IC.

The present study showed that there was no significant difference in efficacy between the first and second HD/FUL. Rather, the maximum bladder capacity at the second HD/FUL was smaller than that at the first HD/FUL, although this difference was not significant. Ottem and Teichman [3] demonstrated that patients undergoing their first versus a repeated cystoscopy with hydrodistension had a hydrodistension volume of $772 \pm 138 \mathrm{~mL}$ versus $646 \pm 177 \mathrm{~mL}(\mathrm{P}=0.02)$, while the median duration of responders was not significantly different between the first and repeat therapies $(\mathrm{P}=0.99)$. Similarly, Ko et al. [8] reported that, in Hunner type IC, there was no significant difference in recurrence-free time between the first and second ablation for Hunner lesions. These results support our finding that the difference in postoperative status between the first and second HD/FUL had no effect on the efficacy of maintenance DMSO therapy.

Currently, several reports have examined the efficacy of combination therapy for improving the short-term efficacy following therapeutic endoscopic treatment. Kuo and Chancellor 
[4] reported that intravesical injections of botulinum toxin type A followed by hydrodistention produced significantly better clinical results than hydrodistention alone in IC patients. Lee and Kuo [23] also demonstrated that repeated intravesical injections of onabotulinumtoxinA were safe, effective, and sustainable in the treatment of IC patients who did not respond to conventional treatment. Shao et al. [9] reported that intravesical instillation of hyaluronic acid may prolong the effect of bladder hydrodistention in patients with severe IC. The present study demonstrated the clinical benefit of using DMSO to sustain the therapeutic efficacy of endoscopic treatment in patients with Hunner lesions.

This study has several limitations. First, this was a pilot study with a small population and analysis conducted using an intrasubject control. As this study was a single-arm trial with no placebo-arm, we cannot deny the possibility of having a placebo effect. Clinical applicability should be further evaluated in a randomized, double-blind, parallel-group, placebo-controlled study. On the other hands, as DMSO has a strong garlic odor, there may be an inherent problem to perform placebo-controlled trials. Second, we defined therapeutic failure as the need to receive repeat endoscopic treatment or other intravesical instillation therapy due to the recurrence of IC symptoms. This definition was based solely on subjective symptoms; therefore, there may have been biases in judging the timing of the second HD/FUL or failure of maintenance DMSO therapy. However, because the symptoms of IC varied from patient to patient, it was difficult to centrally define therapeutic failure using the symptom score or other clinical variables. In fact, several previous reports have defined therapeutic failure based on subjective symptoms $[4,7,23]$. Third, the length of time for which maintenance DMSO therapy should be continued in cases without recurrence of IC symptoms is controversial. Our results demonstrated that patients who continued maintenance DMSO therapy for more than 5 years showed no subsequent recurrence of IC symptoms. However, future prospective studies should verify the timing of treatment discontinuation.

In conclusion, therapeutic endoscopic treatment plus maintenance DMSO therapy clearly prolongs the recurrence-free time compared with therapeutic endoscopic treatment alone in Hunner type IC. A randomized, double-blind, parallel-group, placebo-controlled study should be performed to confirm our results.

\section{AUTHOR CONTRIBUTION STATEMENT}

- Full access to all the data in the study and takes responsibility for the integrity of the data and the accuracy of the data analysis: $A O$

- Study concept and design: $A O$

- Acquisition of data: AO, TS, YM, HW, KT, DM, TI, TS

- Analysis and interpretation of data: $A O$

- Drafting of the manuscript: $A O$

- Critical revision of the manuscript for important intellectual content: TS, YM, HW, KT, DM, TI, TS, HM

- Statistical analysis: $A O$

- Obtained funding: None

- Administrative, technical, or material support: None

- Study supervision: $H M$

\section{REFERENCES}

1. Homma Y, Ueda T, Tomoe H, Lin AT, Kuo HC, Lee MH, et al. Clinical guidelines for interstitial cystitis and hypersensitive bladder updated in 2015. Int J Urol 2016;23:542-9.

2. Glemain P, Rivière C, Lenormand L, Karam G, Bouchot O, Buzelin JM. Prolonged hydrodistention of the bladder for symptomatic treatment of interstitial cystitis: efficacy at 6 months and 1 year. Eur Urol 2002;41:79-84.

3. Ottem DP, Teichman JM. What is the value of cystoscopy with hydrodistension for interstitial cystitis? Urology 2005;66:494-9.

4. Kuo HC, Chancellor MB. Comparison of intravesical botulinum toxin type A injections plus hydrodistention with hydrodistention alone for the treatment of refractory interstitial cystitis/painful bladder syndrome. BJU Int 2009;104:657-61.

5. Peeker R, Aldenborg F, Fall M. Complete transurethral resection of ulcers in classic interstitial cystitis. Int Urogynecol J Pelvic Floor Dysfunct 2000;11:290-5.

6. Hillelsohn JH, Rais-Bahrami S, Friedlander JI, Okhunov Z, Kashan $\mathrm{M}$, Rosen L, et al. Fulguration for Hunner ulcers: long-term clinical outcomes. J Urol 2012;188:2238-41.

7. Niimi A, Nomiya A, Yamada Y, Suzuki M, Fujimura T, Fukuhara H, et al. Hydrodistension with or without fulguration of hunner lesions for interstitial cystitis: long-term outcomes and prognostic predictors. Neurourol Urodyn 2016;35:965-9.

8. Ko KJ, Chung H, Suh YS, Lee SW, Kim TH, Lee KS. Therapeutic effects of endoscopic ablation in patients with Hunner type interstitial cystitis. BJU Int 2018;121:659-66.

9. Shao Y, Shen ZJ, Rui WB, Zhou WL. Intravesical instillation of hyaluronic acid prolonged the effect of bladder hydrodistention in patients with severe interstitial cystitis. Urology 2010;75:547-50. 
10. Hsieh CH, Chang WC, Huang MC, Su TH, Li YT, Chang ST, et al. Hydrodistention plus bladder training versus hydrodistention for the treatment of interstitial cystitis. Taiwan J Obstet Gynecol 2012; 51:591-5.

11. Rawls WF, Cox L, Rovner ES. Dimethyl sulfoxide (DMSO) as intravesical therapy for interstitial cystitis/bladder pain syndrome: a review. Neurourol Urodyn 2017;36:1677-84.

12. Jacob SW, Bischel M, Herschler RJ. Dimethyl sulfoxide (DMSO): a new concept in pharmacotherapy. Curr Ther Res Clin Exp 1964;6:134-5.

13. Shiga KI, Hirano K, Nishimura J, Niiro N, Naito S, Kanaide H. Dimethyl sulphoxide relaxes rabbit detrusor muscle by decreasing the Ca2+ sensitivity of the contractile apparatus. Br J Pharmacol 2007; 151:1014-24.

14. Santos NC, Figueira-Coelho J, Martins-Silva J, Saldanha C. Multidisciplinary utilization of dimethyl sulfoxide: pharmacological, cellular, and molecular aspects. Biochem Pharmacol 2003;65:1035-41.

15. Birder LA, Kanai AJ, de Groat WC. DMSO: effect on bladder afferent neurons and nitric oxide release. J Urol 1997;158:1989-95.

16. Wein A, Hanno P, Gillenwater J. Interstitial cystitis: an introduction to the problem. London: Springer-Verlag; 1990.

17. Ek A, Engberg A, Frödin L, Jönsson G. The use of dimethyl-sulfoxide (DMSO) in the treatment of interstitial cystitis. Scand J Urol Nephrol 1978;12:129-31.
18. Peeker R, Haghsheno MA, Holmäng S, Fall M. Intravesical bacillus Calmette-Guerin and dimethyl sulfoxide for treatment of classic and nonulcer interstitial cystitis: a prospective, randomized doubleblind study. J Urol 2000;164:1912-5.

19. Tomoe H. In what type of interstitial cystitis/bladder pain syndrome is DMSO intravesical instillation therapy effective? Transl Androl Urol 2015;4:600-4.

20. Hanno PM, Burks DA, Clemens JQ, Dmochowski RR, Erickson D, Fitzgerald MP, et al. AUA guideline for the diagnosis and treatment of interstitial cystitis/bladder pain syndrome. J Urol 2011;185:216270.

21. Hanno PM, Erickson D, Moldwin R, Faraday MM; American Urological Association. Diagnosis and treatment of interstitial cystitis/ bladder pain syndrome: AUA guideline amendment. J Urol 2015;193:1545-53.

22. Lee SW, Kim WB, Lee KW, Kim JM, Kim YH, Lee B, et al. Transurethral resection alone vs resection combined with therapeutic hydrodistention as treatment for ulcerative interstitial cystitis: initial experience with propensity score matching studies. Urology 2017;99:62-8.

23. Lee CL, Kuo HC. Long-term efficacy and safety of repeated intravescial onabotulinumtoxinA injections plus hydrodistention in the treatment of interstitial cystitis/bladder pain syndrome. Toxins (Basel) 2015;7:4283-93. 